Supporting Information for

\title{
In Situ Visualization of Lithium Penetration through Solid Electrolyte and Dead Lithium Dynamics in Solid-State Lithium Metal Batteries
}

Haiming Sun $,{ }^{\dagger}, \mid$ Qiunan Liu,${ }^{\dagger}$ Jingzhao Chen,${ }^{\dagger}$ Yanshuai $\mathrm{Li},{ }^{\dagger}$ Hongjun Ye, ${ }^{\dagger}$ Jun Zhao,,${ }^{\dagger}$ Lin Geng, ${ }^{\dagger}$ Qiushi Dai,${ }^{\dagger}$ Tingting Yang,${ }^{\dagger}$ Hui Li,${ }^{\dagger}$ Zaifa Wang, ${ }^{\dagger}$ Liqiang Zhang, $,{ }^{\dagger}, *$ Yongfu Tang, $, \stackrel{+}{\star}, *$ and Jianyu Huang ${ }^{\dagger}, \S, *$

$\nmid$ Clean Nano Energy Center, State Key Laboratory of Metastable Materials Science and Technology and $*$ Hebei Key Laboratory of Applied Chemistry, School of Environmental and Chemical Engineering, Yanshan University, Qinhuangdao 066004, P. R. China.

$\S$ School of Materials Science and Engineering, Xiangtan University, Xiangtan, Hunan 411105, P. R. China.

" Research Center for Ultra-High Voltage Electron Microscopy, Osaka University, Ibaraki, Osaka 567-0047, Japan.

Corresponding Author

*Correspondence to: lqzhang@ysu.edu.cn; tangyongfu@ysu.edu.cn; jyhuang8@hotmail.com. 


\section{Table of Contents}

Supplementary Discussion

Supplementary Movies S1-S6 Captions

Supplementary Figures S1-S7

Supplementary References 


\section{Supplementary Discussion}

\section{Effect of the $\mathrm{CO}_{2}$ Gas Environment on Li Deposition}

In our experiment, a thin layer of $\mathrm{Li}_{2} \mathrm{CO}_{3}$ formed on the dendrites surface in the $\mathrm{CO}_{2}$ atmosphere through the reduction of $\mathrm{CO}_{2}$ via: $\mathrm{Li}+\mathrm{CO}_{2} \rightarrow \mathrm{Li}_{2} \mathrm{CO}_{3}+\mathrm{C}$ or CO. ${ }^{1-3}$ The formation of a thin layer of $\mathrm{Li}_{2} \mathrm{CO}_{3}$ on the $\mathrm{Li}$ surface is very similar to the formation

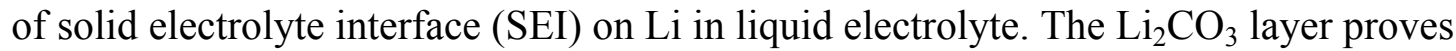
to be essential to stabilize the freshly deposited Li dendrites and permit in situ TEM observation of its penetrating through or lifting up LLZTO slices under electron beam illumination. However, the presence of $\mathrm{CO}_{2}$ does not affect the Li penetration through LLZTO as proved by the following.

To highlight the effect of $\mathrm{CO}_{2}$, a control experiment without $\mathrm{CO}_{2}$ was carried out. As shown in Figure S6, upon applying a negative potential, two dendrites marked as "1" and "2" (Figure S6b) emerged on the top surface of LLZTO, which then grew with lapse of time (Figure S6c). Note that a thin surface layer, most likely $\mathrm{Li}_{2} \mathrm{O}$ due to freshly deposited Li reacting with the residue oxygen in the TEM column, ${ }^{4}$ formed accompanying with the dendrite growth. The surface of the dendrites seems to be more rough compared with that obtained in a $\mathrm{CO}_{2}$ atmosphere. Moreover, Li deposited in vacuum is usually plate-like rather than a dendrite. Also, Li deposited in vacuum tends to deform and even collapse under electron beam imaging (Figure S6d), similar to previous report. ${ }^{3}$ We believe that the presence of $\mathrm{CO}_{2}$ plays a critical role to protect the newly formed $\mathrm{Li}$ dendrites from electron beam damage, and the presence a thin $\mathrm{Li}_{2} \mathrm{CO}_{3}$ layer on the Li surface facilitates the formation of $\mathrm{Li}$ dendrite rather than Li plate. However, Figure S6 shows that Li penetration through LLZTO is not influenced by the $\mathrm{CO}_{2}$ gas. Therefore, without the $\mathrm{CO}_{2}$ atmosphere, we were unable to obtain stable $\mathrm{Li}$ dendrites for observation; Under the effect of beam irradiation, the freshly deposited $\mathrm{Li}$ would be destroyed quickly and left lots of "dead" Li behind. Thus, the $\mathrm{CO}_{2}$ gas environment in the ETEM plays a critical role in the formation of the passivation $\mathrm{Li}_{2} \mathrm{CO}_{3}$ layer to stabilize the $\mathrm{Li}$ dendrites. 


\section{Effect of Electron Beam Irradiation}

In order to investigate the influence of electron beam, a new experiment under the beam blank condition was conducted. The TEM images before and after Li deposition are shown in Figure S7a and $\mathbf{b}$, respectively. At the end of the discharge process, a Li dendrite was formed on the top surface of LLZTO, and many black nanoparticles (marked with yellow arrows) were carried out by Li deposition. The result shows that even without electron beam, we can also observe the penetration of Li dendrites through the LLZTO slice. The results indicate that although the electron beam has an unavoidable effect on Li deposition experiments, however, during our experiments, the dose rate was well controlled to minimize the beam effect. Under our experimental condition, we do not observe Li deposition on LLZTO under only electron beam irradiation but without biasing. Therefore, we have no doubt that the driving force for Li dendrites penetration was the electrochemical potential rather than the electron beam.

Furthermore, the LLZTO slice after Li penetration was transferred into FIB, then the bottom surface of the LLZTO was carefully cleaned and slowly milled toward to the top surface, as shown in Figure S7d. A few cracks can be seen, some of which in the proximity of the Li dendrite (Figure S7b). While in the pristine LLZTO bottom surface, no such cracks were observed (Figure S7a and c). We believe that these cracks serve as the Li nucleation and propagation channel. Li is very likely nucleated from the pre-existed mini-cracks, grain boundaries or voids in the LLZTO, as proposed in several previous publications. ${ }^{5-8}$ However, it is very difficult to directly observe the nucleation process due to the buried interface. 


\section{Supplementary Movies}

\section{Description of Movie S1}

An in situ TEM movies showing the penetration of Li dendrite through LLZTO and the formation of "dead" Li after stripping. The movie was recorded 2 frames per second and played at $48 \times$ speed. (Corresponding to Figure 1c-o)

\section{Description of Movie S2}

An in situ TEM movies showing the penetration of Li dendrite through LLZTO and the formation of "dead" Li after stripping. The movie was recorded 2 frames per second and played at $133 \times$ speed. (Corresponding to Figure 2a-i)

\section{Description of Movie S3}

An in situ TEM movies showing the Li dendrites stripping process and the formation of "dead" Li. The movie was recorded 2 frames per second and played at $10 \times$ speed. (Corresponding to Figure 3)

\section{Description of Movie S4}

An in situ TEM movies showing the penetration of Li dendrite through LLZTO and the formation of "dead" Li after stripping. The movie was recorded 2 frames per second and played at $180 \times$ speed. (Corresponding to Figure S2 and Figure 4)

\section{Description of Movie S5}

An in situ TEM movies showing a growing Li dendrite lifting up LLZTO and the formation of "dead" Li after stripping. The movie was recorded 2 frames per second and played at $20 \times$ speed. (Corresponding to Figure 5a-f)

\section{Description of Movie S6}


An in situ TEM movies showing a growing Li dendrite lifting up LLZTO and the formation of "dead" Li after stripping. The movie was recorded 2 frames per second and played at $11 \times$ speed. (Corresponding to Figure 5g-k) 


\section{Supplementary Figures}

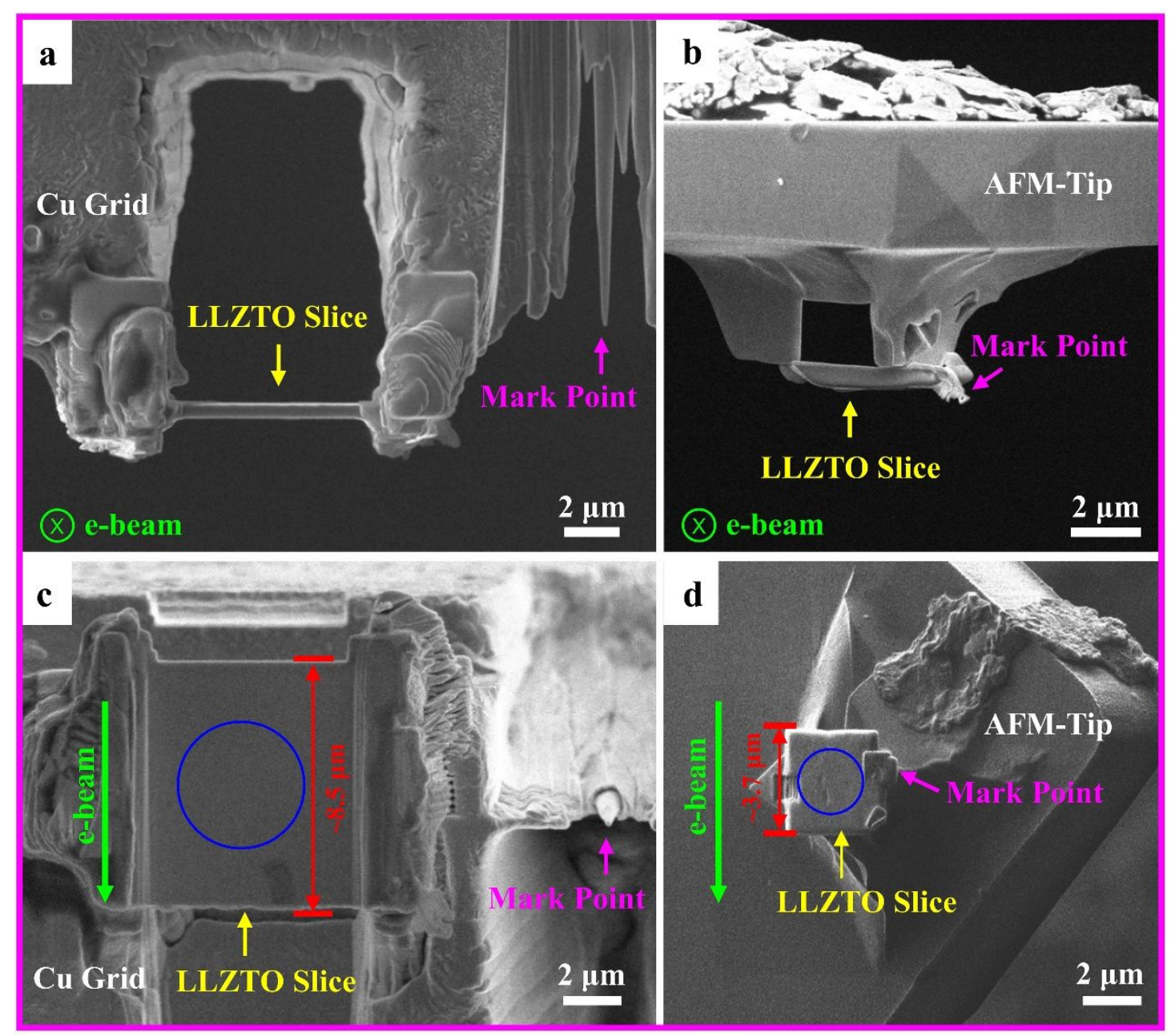

Figure S1 A LLZTO slice attached on the $\mathrm{Cu}$ grid with a width (parallel to e-beam path) of $\sim 8.5 \mu \mathrm{m}$ : (a) side view, (c) bottom view. A LLZTO slice attached on the AFM tip with a width (parallel to e-beam path) of $\sim 3.7 \mu \mathrm{m}$ : (b) side view, (d) bottom view. Blue circles in (c-d) denote the contact region between LLZTO and $\mathrm{Li}_{2} \mathrm{CO}_{3} / \mathrm{Li}$ anode. 


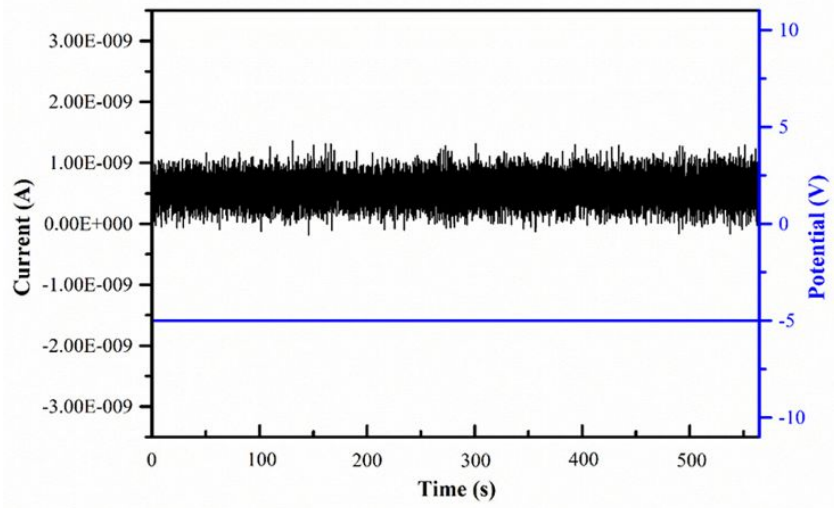

Figure S2 Recorded current and applied potential during Li deposition as shown in Figure 1d-g. 


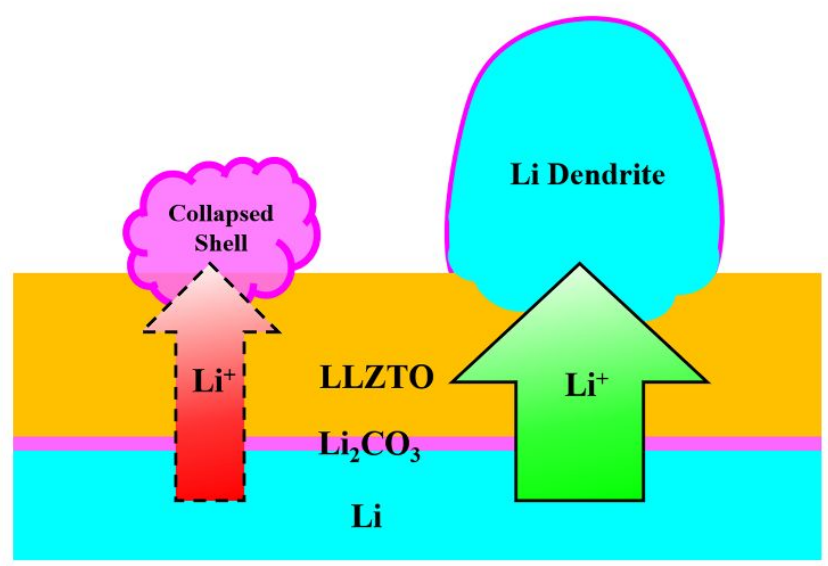

Figure S3 Schematic for the growth of new Li dendrites. 


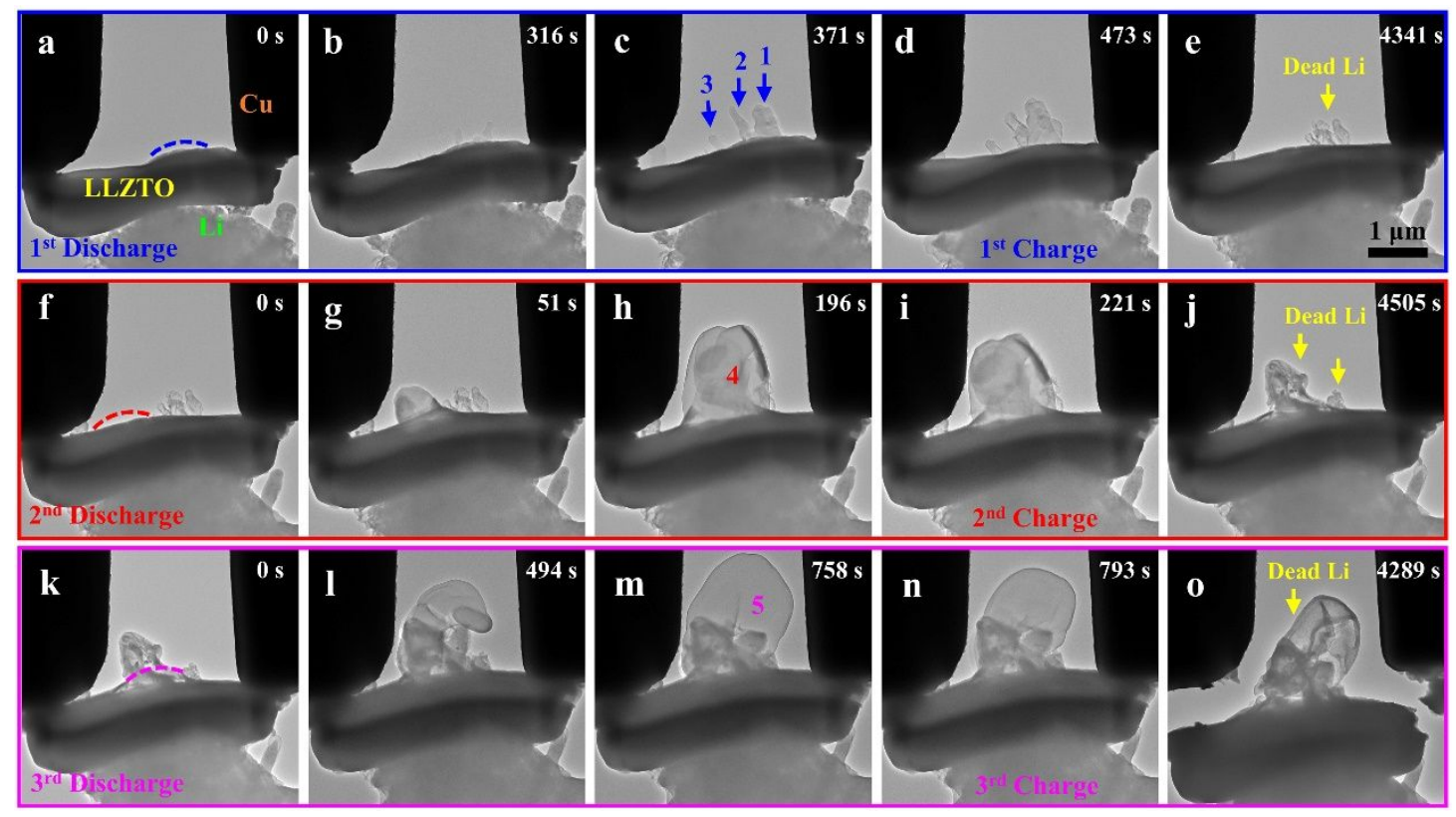

Figure S4 Time-lapse TEM images showing Li dendrite penetration through LLZTO in a $\mathrm{CO}_{2}$ ambient. The numbers "1"-"5" mark the appearance of different dendrites during repeated $\mathrm{Li}$ deposition and stripping. The $1^{\text {st }}$ cycle Li deposition and stripping are shown in (a-c) and (d-e), respectively; the $2^{\text {nd }}$ Li deposition and stripping are shown in (f-h) and (i, j), respectively; the $3^{\text {rd }} \mathrm{Li}$ deposition and stripping are shown in (k-m) and (n, o), respectively. (Corresponding to Movie S4) 

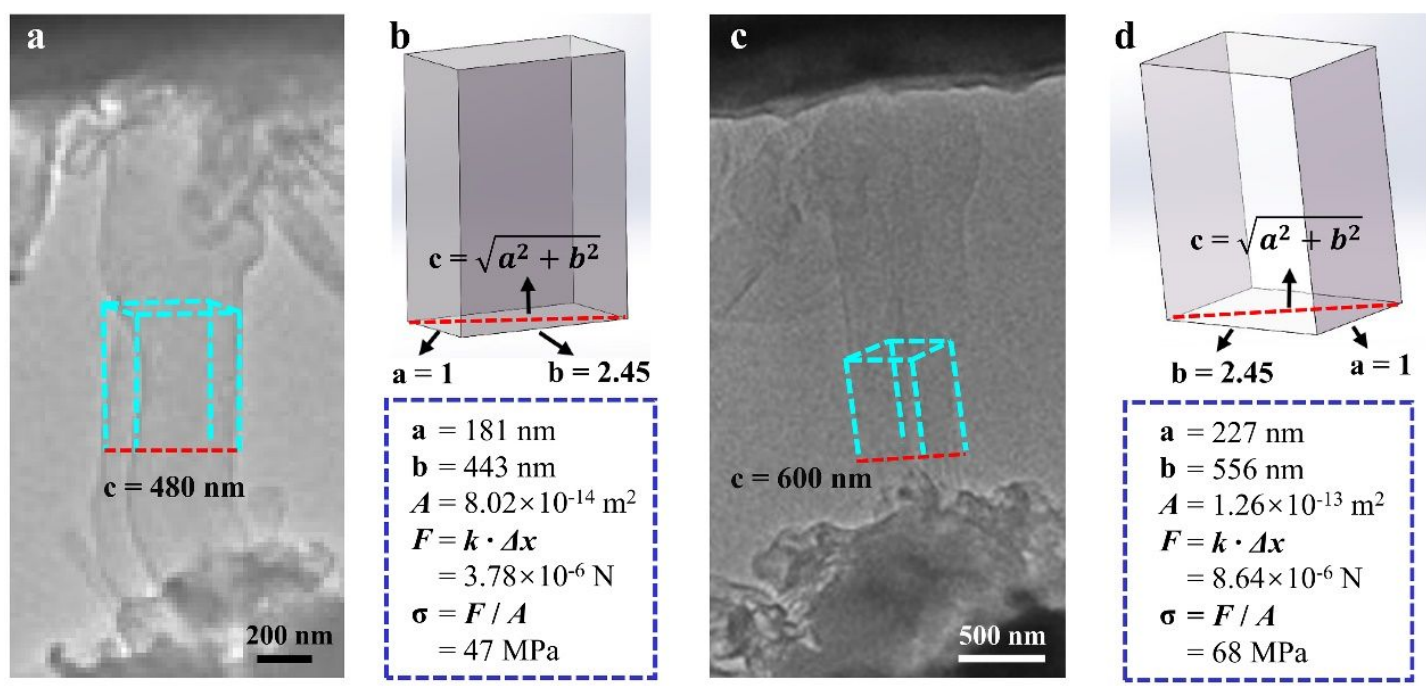

Figure S5 (a, c) The cross-section of the dendrites is rectangular. (b, d) Schematic of the geometry of the dendrite, on which the cross-section area calculation is based. 


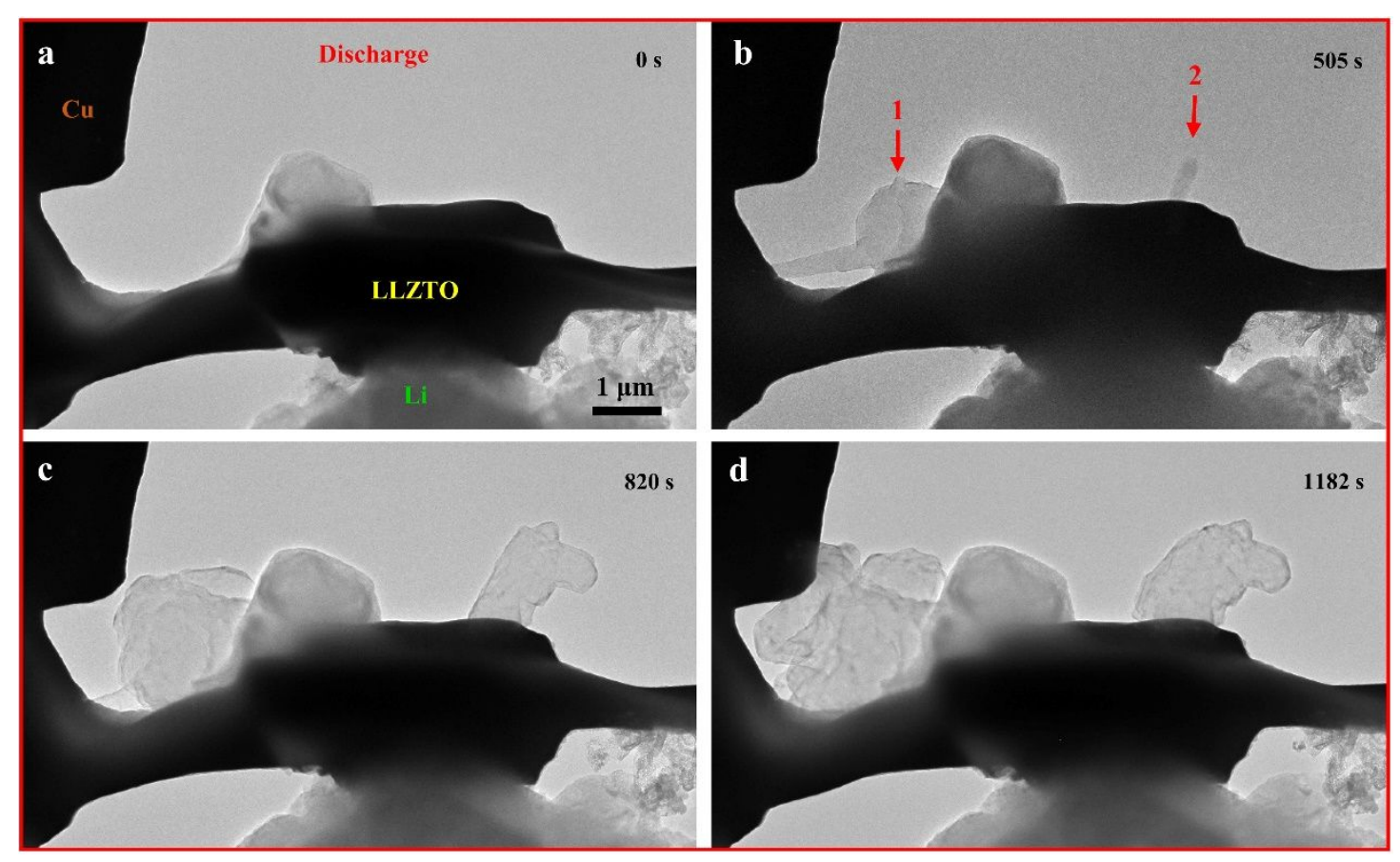

Figure S6 (a-d) Time-lapse TEM images showing Li dendrite penetration through LLZTO in a high vacuum ambient. 


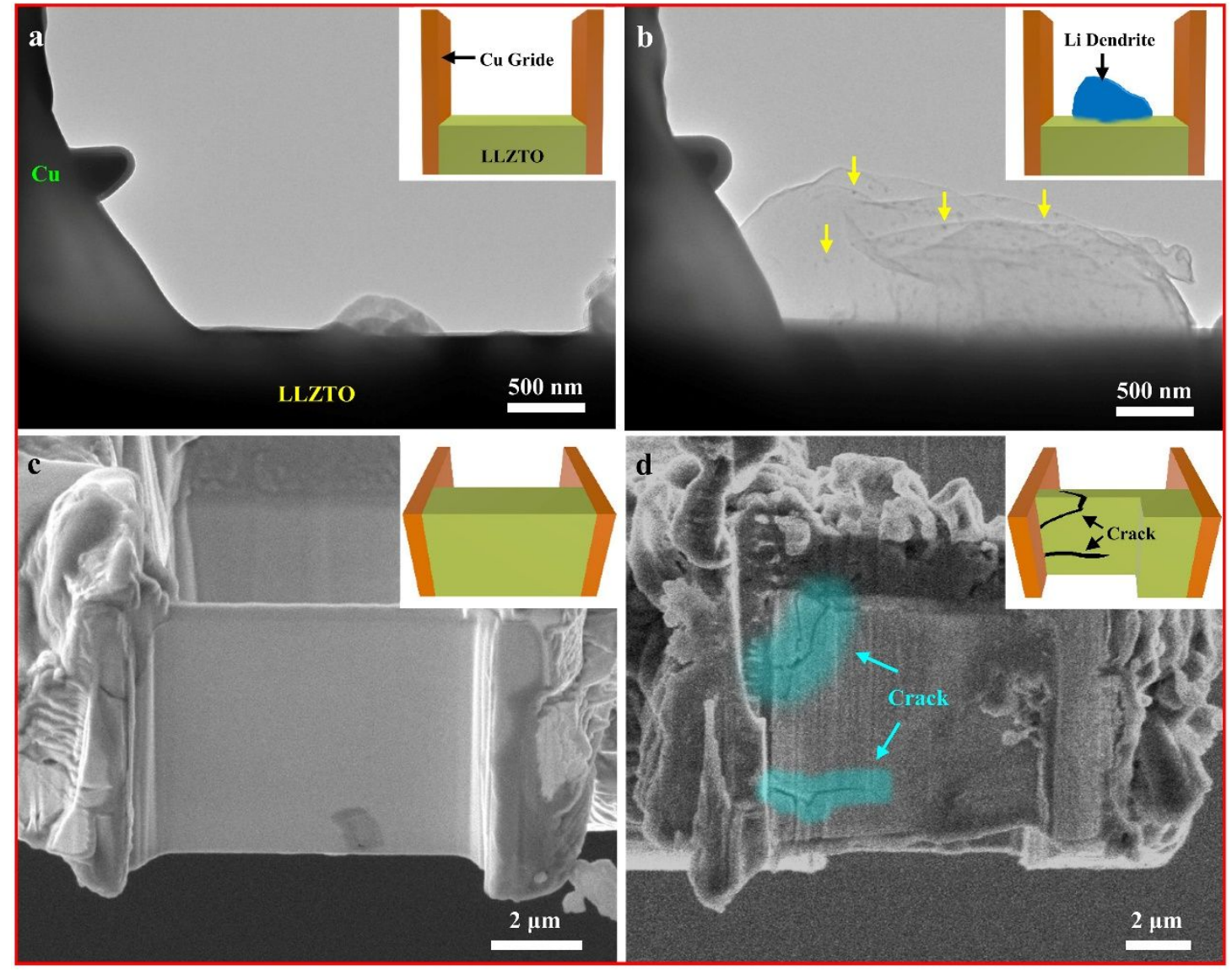

Figure S7 (a, b) TEM images of the LLZTO slices before (a) and after (b) Li dendrites' penetration. (c) SEM image (bottom view) of the pristine LLZTO slice. (d) SEM image of the LLZTO after Li dendrites' penetration and FIB milling, showing presence of cracks (the cyan-shaded regions) caused by Li deposition. Insets are schematics of the experimental configuration. 


\section{Supplementary References}

(1) Xu, S.; Das, S. K.; Archer, L. A. The Li-CO $\mathrm{CO}_{2}$ Battery: A Novel Method for $\mathrm{CO}_{2}$ Capture and Utilization. RSC Adv. 2013, 3, 6656-6660.

(2) Yang, T.; Li, H.; Tang, Y.; Chen, J.; Ye, H.; Wang, B.; Zhang, Y.; Du, C.; Yao, J.; Guo, B.; Shen, T.; Zhang, L.; Zhu, T.; Huang, J. In Situ Observation of Cracking and Self-Healing of Solid Electrolyte Interphases during Lithium Deposition. Sci. Bull. 2021, 66, 1754-1763.

(3) Zhang, L.; Yang, T.; Du, C.; Liu, Q.; Tang, Y.; Zhao, J.; Wang, B.; Chen, T.; Sun, Y.; Jia, P.; Li, H.; Geng, L.; Chen, J.; Ye, H.; Wang, Z.; Li, Y.; Sun, H.; Li, X.; Dai, Q.; Tang, Y.; et al. Lithium Whisker Growth and Stress Generation in an in Situ Atomic Force Microscope-Environmental Transmission Electron Microscope Set-Up. Nat. Nanotechnol. 2020, 15, 94-98.

(4) Zheng, H.; Liu, Y.; Mao, S. X.; Wang, J.; Huang, J. Y. Beam-Assisted Large Elongation of in Situ Formed $\mathrm{Li}_{2} \mathrm{O}$ Nanowires. Sci. Rep. 2012, 2, 542.

(5) Wu, B.; Wang, S.; Lochala, J.; Desrochers, D.; Liu, B.; Zhang, W.; Yang, J.; Xiao, J. The Role of the Solid Electrolyte Interphase Layer in Preventing Li Dendrite Growth in Solid-State Batteries. Energy Environ. Sci. 2018, 11, 1803-1810.

(6) Han, F.; Westover, A. S.; Yue, J.; Fan, X.; Wang, F.; Chi, M.; Leonard, D. N.; Dudney, N. J.; Wang, H.; Wang, C. High Electronic Conductivity as the Origin of Lithium Dendrite Formation within Solid Electrolytes. Nat. Energy 2019, 4, 187196.

(7) Liu, X.; Garcia-Mendez, R.; Lupini, A. R.; Cheng, Y.; Hood, Z. D.; Han, F.; Sharafi, A.; Idrobo, J. C.; Dudney, N. J.; Wang, C.; Ma, C.; Sakamoto, J.; Chi, M. Local Electronic Structure Variation Resulting in Li 'Filament' Formation within Solid Electrolytes. Nat. Mater. 2021, https://doi.org/10.1038/s41563-021-01019$\mathrm{x}$.

(8) Cheng, E. J.; Sharafi, A.; Sakamoto, J. Intergranular Li Metal Propagation through Polycrystalline $\mathrm{Li}_{6.25} \mathrm{Al}_{0.25} \mathrm{La}_{3} \mathrm{Zr}_{2} \mathrm{O}_{12}$ Ceramic Electrolyte. Electrochim. Acta 2017, 223, 85-91. 